Hora, H., et al. Low Energy Nuclear Reactions resulting as picometer interactions with similarity to Kshell electron capture. in Eleventh International Conference on Condensed Matter Nuclear Science. 2004. Marseille, France.

\title{
Low Energy Nuclear Reactions resulting as picometer interactions with similarity to K-shell electron capture
}

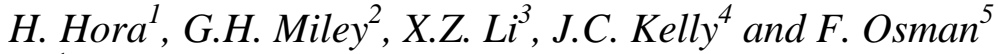 \\ ${ }^{1}$ University of New South Wales, Sydney, 2052, Australia \\ ${ }^{2}$ Fusion Studies Laboratory, University of Illinois 61801, Urbana, IL, USA \\ ${ }^{3}$ Physics Department, Tsinghua University, Beijing 100084, China \\ ${ }^{4}$ School of Physics, Sydney University, Sydney 2006, Australia \\ ${ }^{5}$ University of Western Sydney, Penrith-South, NSW 1791, Australia
}

Since the appeal by Brian Josephson at the meeting of the Nobel Laureates July 2004, it seems to be indicated to summarize the following serious, reproducible and confirmed observations on reactions of protons or deuterons incorporated in host metals such as palladium. Some reflections to Rutherford's discovery of nuclear physics, the CockroftOliphant discovery of anomalous low energy fusion reactions and the chemist Hahn's discovery of fission had to be included. Using gaseous atmosphere or discharges between palladium targets, rather significant results were seen e.g. from the "life after death" heat production of such high values per host atom that only nuclear reactions can be involved. This supports the earlier evaluation of neutron generation in fully reversible experiments with gas discharges hinting that a reasonable screening effect - preferably in the swimming electron layer - may lead to reactions at nuclear distances $d$ of picometers with reaction probability times $U$ of about megaseconds similar to the K-shell capture radioactivity. Further electrolytic experiments led to Low Energy Nuclear Reactions (LENR) where the involvement of pollution could be excluded from the appearance of very seldom rare earth elements. A basically new theory for DD cross sections is used to confirm the picometer-megasecond reactions of cold fusion. Other theoretical aspects are given from measured heavy element distributions similar to the standard abundance distribution, $\mathrm{SAD}$, in the Universe with consequences on endothermic heavy nuclei generation, magic numbers and to quark-gluon plasmas.

\section{1) Prelude and Introduction}

The public opinion and the view of the media is still uncertain about the physics phenomenon of cold fusion or the low energy nuclear reactions, LENR, which may occur by high concentration of protons or deuterons in host metals as palladium, nickel and others. There should be no doubt in physics in clarifying truth and only one truth, and even if non-physicists have different tools or views to address these questions, a strange situtation has developed since 1989. For physicists, a list for clearly formulated questions has been presented by Brian Josephson [1] from which position at least a starting point for clarification should be possible. 
The problem may be that the phenomena were brought forward to physics by nonphysicists. In this situation it may be permitted to recapitulate what happened in similar cases before. When Becquerel discovered 1896 that the pitchblende from St. Joachimstal in Bohemia and other minerals containing uranium are emitting certain radiation blackening photographic plates, a wide range of people were speculating about this phenomenon. There were even papers explaining that some ghosts are involved. Ernest Rutherford after his undergraduate studies in New Zealand produced splendid results with his Ph.D. in Cambridge where he before 1900 contributed to Marconi's detection of electromagnetic radiation by discovering very ingeniously the "radiomagnetic detectors". After becoming a professor at the McGill University in Montreal, he discovered that pitchblende emitted helium as demonstrated spectroscopically and found that another emission were energetic electrons which were just recognized at this time. With this discovery of alpha- beta- and gamma-radiation he became the founder of nuclear physics but his faculty was going to dismiss him because he was working in a field related to ghosts. He was saved in last minute by the offer of a professorship in England.

After Rutherford discovered that the atoms are empty and there was only a very tiny nucleus in the center and that these nuclei could interact with others if they were bombarded with particles of several million electron volt energy to overcome the Coulomb repulsion, $\mathrm{MeV}$ accelerators were built and a broad field of nuclear reactions was studied. It was then the idea by Cockroft [2] that he used his multi-MeV accelerator to bombard light nuclei with light nuclei at energies 50 times lower against all expectations and opinions how to overcome the Coulomb repulsion. And there were reactions e.g of protons with ${ }^{11} \mathrm{~B}$. At this stage, Mark Oliphant who had come from South Australia for his Ph.D. to Rutherford in Cambridge, used his very powerful 100 kilovolt gas discharges for these nuclear bombardments and received the precise energy for the $\mathrm{p}$ $\mathrm{B}(11)$ reaction [3]. Then he tried to use the not long before discovered heavy hydrogen deuterium D instead of the protons in his discharge [4] - produced by methods braught over by Paul Harteck from Germany - and found reactions with traces in the cloud chamber where even the grand master of this field, Lord Rutherford could not find an answer for several days. But then it was clear: the D-D reaction even working at $10 \mathrm{keV}$ and less was producing the then not known superheavy hydrogen isotope tritium $\mathrm{T}$ and the very rare ${ }^{3} \mathrm{He}$ isotope with several branches of reactions unknown before. This first nuclear fusion reaction in 1933/34 is now a wide field for energy research.

When few years before the deuterium was available in Berlin, Paneth tried to see what happens when this heavy hydrogen was used in the long known phenomenon of very high concentration (even above 1:1) absorbed in palladium at room temperature, it seemed that helium was emitted [2] - a strange analogy to the pitchblende. Physicist were sceptical and Paneth had to withdraw [6]. The incorporation of hydrogen or of its isotopes in palladium was indeed remarkable and this was used for transporting of protons or its isotopes through palladium layers which had to contain a small percentage of silver in vacuum techniques. The strong decrease of radioactivity when loading tritium $\mathrm{T}$ in $\mathrm{Ti}$ was noticed also in such a prestigious laboratory as that of Philips in Eindhoven [7] and neutron emission was reported from palladium compounds [8]. End of 1989 it was reported from the BARC (Bhabha Atomic Research Laboratory) where the Indians developed their nuclear weapons, that when moving $\mathrm{D}$ through $\mathrm{Pd}$, tritium was appearing on the rare side as measured with their necessarily very sensible tritium detectors. $\mathrm{M}$. 
Srinivasan reported that there were samples of Pd containing D stored for 15 years which then showed tritium in dangerous quantities which definitely had not been incorporated 15 years before. When Gopal Ayengar, Director of BARC, reported this to the top researchers at the Kurchatov institute in Moscow, they responded with icy tacite only.

As a reaction to the reports on anomalous heat production from D loaded Pd April 1989 at the University of Utah [9] and neutron emission [10], the Kurtchatov institute like many other places liked to reproduce the reported anomalies but without success. S. Pismeny (Director of the Troitsk branch of the Kurtchatov Institute) mentioned that the money given to Fleischmann [9] was used mostly to rebuilt a large number of the initial electrolytic cells which could not demonstrate more than what was questionable in the beginning. Dozens of Million dollars were spent from Japan for research in a private laboratory in France and in a government laboratory in Sapporo. The latter one bought a large number of these electrolytic cells from France where its was claimed that these produced heat. When in Sapporo, no heat appeared even after Pons was there for several weeks working with the cells as M. Okamoto reported.

Even physicists have examples where such failure in reproducing claimed observations do happen, before the complexity of a new situation in physics is clarified. The more sceptical are physicists when chemists or others are claiming anomalies in physics. Such a problem was between the chemist Otto Hahn and the radiation physicist Lise Meitner, a most prominent team e.g. with the discovery of the new element protactinium in 1918 where they - against the rules - did not receive the Nobel prize. Physicists expected the production of heavier nuclei when bombarding uranium with neutrons into which direction Meitner was looking when she left Berlin mid 1938 under unfavourable circumstances to Stockholm. Meitner meeting Hahn November 1938 [11] still "objected to the most recent findings" of Hahn who then again with his world best techniques of chemical micro-analysis confirmed that elements of middle weight were produced, proving that the neutrons were splitting the uranium nucleus. These results were reproduced very quickly in comparably easy experiments and the enormous consequences are known.

In support of the arguments of Josephson [1] we are trying to report on reproducible results which physicists may consider worthwhile to re-examine. We are aware that the last 15 years produced more than $95 \%$ publications which cannot be accepted by physicists beginning with the theory that gravitation waves form far out galaxies cause cold fusion, not to talk about the ghosts with the Becquerel radiation. Since every coldfusionist likes to get his own credit, nearly nobody is taking the work of colleagues serious or carefully reproduces the other's work. It is really the responsibility and duty of funding agencies like DOE or others to financially attract most carefully selected teams to reproduce the one or the other serious result as the very first step. Only after this clarifying progress on experiments, one may talk about any theory or model [14]. Neverthelss - indeed with all reservations - we are discussing some theoretical aspects in the following. Using the first complete theory about hot fusion cross sections [9.10] based on a complex Schrödinger potential, we show direct agreement with the results of the picometer-megasecond nuclear reaction model which was concluded earlier from experimental results. 


\section{2) Experimental Facts}

Most of the reported observations of heat generation or radiation emission from deuterium loaded palladium are occurring not regularly, are pulsating statistically and not reproducible. This was summarized by Yamaguchi et al [15] and distinguished from the few reproducible observations of neutron and gamma emission where the palladium was in a gaseous environment and where gas discharges were used and complications with electrolytic procedures were avoided. One of the reproducible results were that by Prelas et al [16]. In this case it was noted that the standard cleaning of the palladium surface by an argon pre-discharge was essential and the interaction with air stopped the neutron emission indicating that the surface conditions of the palladium are important.

The generation of heat was indeed in the focus of interest. Experiments were performed in gaseous atmosphere at different pressures and temperatures placing $\mathrm{Pd}$ wires in deuterium gas [17] but observing also effects if not deuterium but light hydrogen is loaded into the palladium [18]. Long time repeated experiments with $\mathrm{Pd}$ wires in hydrogen atmosphere showed "heat after death": heat was generated after the gas loading discharge had been stopped and the gas was evacuated. For the following 43 hours, the wires produced $3.6 \mathrm{~kW} / \mathrm{cm}^{3}$ heat or $13 \mathrm{keV}$ per palladium atom. Such energy cannot be produced by chemical processes. Since any heat generating process will not be due to every average $\mathrm{Pd}$ atom but to specific ones only, reactions with the well known $\mathrm{MeV}$ can be concluded as expected from nuclear reactions. This experiment [18] was repeated at different places and witnessed by experts from the MIT.

If such nuclear reactions occure - even if without emission of alphas, betas, neutrons or not resulting in radioactive reaction products - the $\mathrm{MeV}$ recoil of daughter nuclei should produce $\mathrm{x}$-rays in the few $\mathrm{keV}$ range and the $\mathrm{MeV}$ daughter nuclei should appear as traces in CR39 foils. Both has been detected [19], the in-situ X-rays and the charged particles using the evaluation of the CR39 foils at the Dubna Nuclear Research Center. Before it was shown [20] that the CR39 traces differ considerably from that of alpha traces, being larger due to the larger $\mathrm{MeV}$ nuclear reaction products.

A proof that the produced new elements after reaction of hydrogen or deuterium in $\mathrm{Pd}$ are not contamination from walls etc., can be seen from the fact, that rarest of rare earths nuclei were generated, e.g. terbium [21] as detected uniquely from the K-shell x-ray spectrum.

There are many more experimental results which, however, need more careful repetition or more accurate measurement before convincing arguments can be established. In the following sections some results are compared with some modelling or consistency proofs as examples how further research may be directed.

\section{3) Modelling nuclear interactions at picometer distance}

Though the DD reaction in palladium is not fully explored experimentally by not fully clarified weighting of branches leading to tritium, neutron production and directly to ${ }^{4} \mathrm{He}$, the reproducible results of the continuous generation of neutrons (in contrast to stochastic bursts [15]) as measured from Pd when loaded with deuterium in gas discharges [16] were used for estimations for fusion reactions. The details of the measurements with 
argon discharge cleaning of the Pd surface, stopping of neutron production when air was let into the reaction chamber and re-establishing of the reaction after argon discharge and

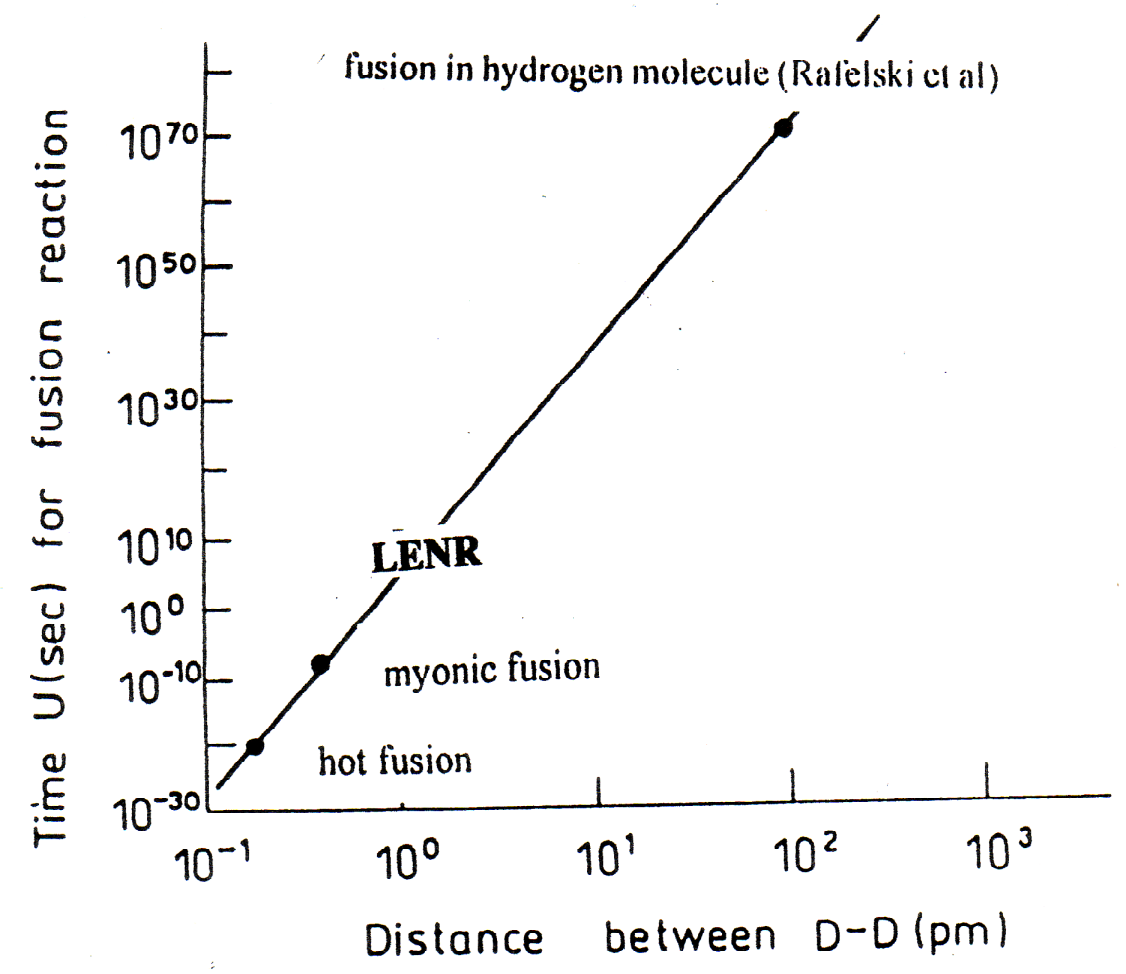

ive confidence to assume

Fig. 1 Measured reaction times $U$ and nuclear distance $d$ in picometers. Points from the left: hot fusion, myonic catalysed fusion and calculated fusion of $D D$ in a $D_{2}$ moleule [22]

The starting point [22] was the mentioned large distance anomaly of the reaction of light nuclei as discovered as an unexpected phenomenon by Cockroft [2] and further clarified by Oliphant et al. [3] leading to the discovery of the very anomalous DD and DT hot fusion reactions [4] with their more than 1000 times larger cross sections than the usual nuclear reactions having mbarn cross sections. The fusion reactions appeared at central collision distance $\mathrm{d}$ with energies $\mathrm{E}$

$$
\mathrm{d}=\mathrm{e}^{2} / \mathrm{E}=1.43 \times 10^{-15} / \mathrm{E}(\mathrm{cm}) \quad(\mathrm{E} \text { in } \mathrm{eV})
$$

at $\mathrm{E}$ of $10 \mathrm{keV}$ or even much less. The distance $\mathrm{d}$ is then $143 \mathrm{fm}$ or larger. This is about hundred times larger than the diameter of the deuterons! Expecting that these hot fusion reactions happen within the usual $10^{-20}$ seconds, and taking the well known reaction time $\mathrm{U}$ for muonic fusion [23], and furthermore taking the estimated reaction time $\mathrm{U}$ for deuterons in a heavy hydrogen molecule [24], a plot of Fig. 1 resulted in a relation [22] 


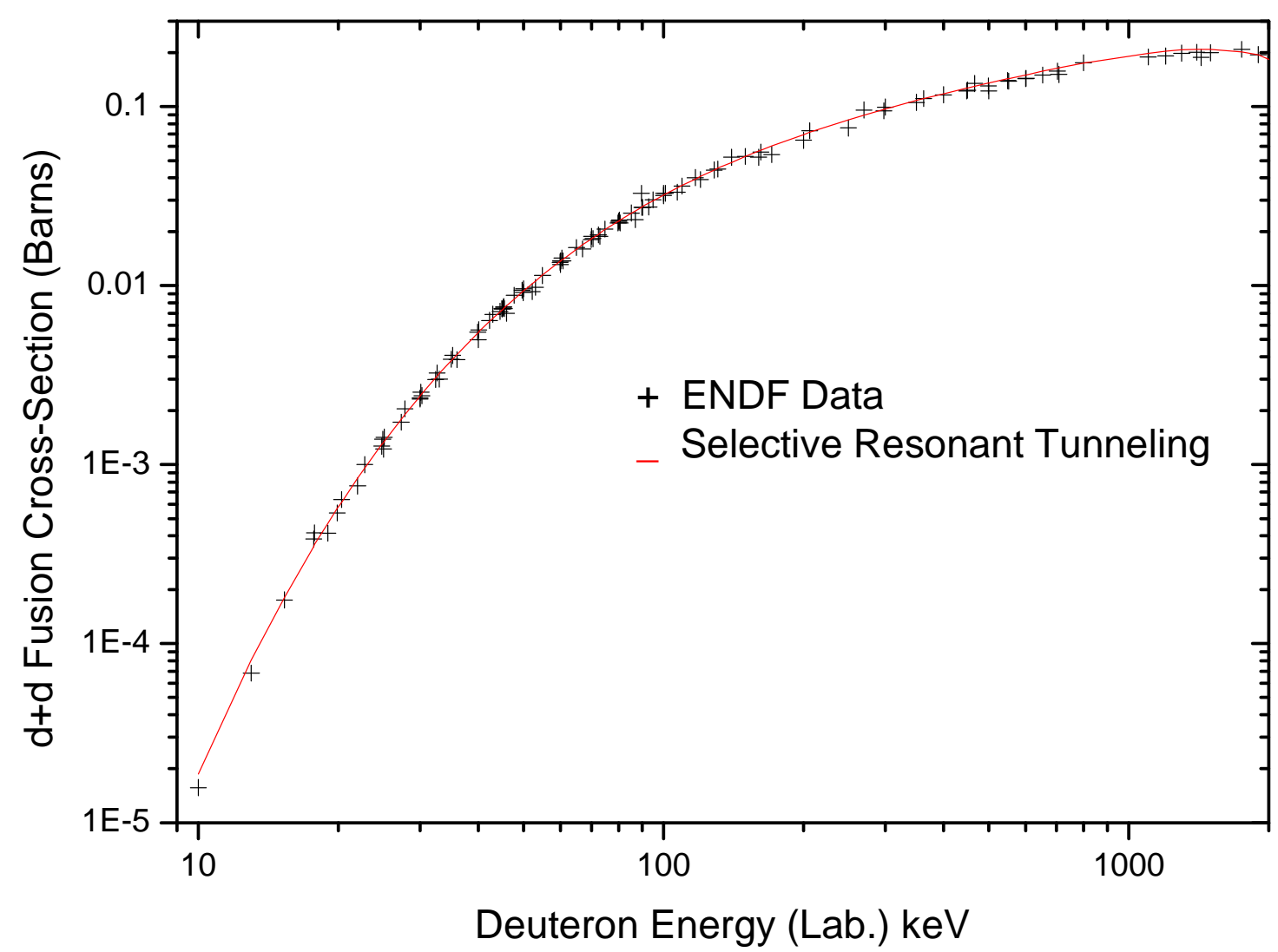

Fig. 2 Comparison between experimental and theoretical calculation for $d+d$ fusion cross-section [13]

$$
\mathrm{U}=8.139 \times 10^{4} \mathrm{~d}^{34.8}(\mathrm{sec}) \quad(\mathrm{d} \text { given in picometer } \mathrm{pm})
$$

The protons or deuterons in the palladium are assumed to be in a state of a Maxwellian gas with a screening $\mathrm{S}$ reducing the Coulomb repulsion as for central collisions to distances $\mathrm{d}$ of

$$
d=(1 / S)^{2} e^{2} / E
$$

the question is still open whether such a strong screening is within the bulk of the Pd or only near the surface due to the well known swimming electron layer [25]. For the interior of high temperature plasma, screenings of $S=5$ are well known [26] and for solid Pd higher values in the interior or especially near the electron layer at the surface may well be possible. When evaluating the reproducible continuos neutron emission from the surface of D loaded Pd [16], a screening S = 14 was estimated [22]. The reaction distance 
of the deuterons is then in the pm range and rather smaller than that of central collision of about 3 pm.

Following the Eq. (2) and Fig. 1, the reactions are then occurring in the range of picometers and with reaction times $U$ of about megaseconds. It should be noted that these times are similar to the measured half life of radioactivity at K-shell electron capture where the Bohr radius is in the similar range of $\mathrm{pm}$.

The picometer reaction distance is in some agreement also with other models [22] for cold fusion. It should be noted that the Debye length for the protons or deuterons at 1:1 loading in $\mathrm{Pd}$ at room temperature is $4.8 \mathrm{pm}$ what may permit the assumption that these ions are moving around within the $\mathrm{Pd}$ nuclei and their electron clouds like neutral particles whose electric charge is cut off a these pm distances permitting the nuclear reactions with any heavy nuclei within the long times of orders of magnitudes of megaseconds.

\section{4) Comparison with new cross section calculations}

The agreement with the measured neutron emission [16] and the screening for picometer DD reactions was possible only for the energetic tail of the Maxwellian distribution of the deuterons in the Pd host metal. It was necessary that the deuterons had to have at least an energy of about $2.4 \mathrm{eV}$ for the reaction. The screening $\mathrm{S}=14$ corresponded then to protons in unscreened low density high temperature plasma of 470 $\mathrm{eV}$ where even for DD a certain very low reaction probability can be expected.

We compare this now with the new theory for the fusion cross sections [12] using a complex Schrödinger potential for light nuclei. All other models for cross sections were numerical fitting of experimental values, e.g. with five constants [27]. The new theory uses only the two reasonable parameters of physics, the resonance energy and the width of the resonance distribution. The model uses a square well nuclear potential and calculates the selective resonant tunnelling. The imaginary part of the potential accounts for the absorption insided the nuclear well. This optical nuclear model was used before for heavy nuclei only. The resonant tunnelling is usually treated as a tow-step process with decay independent tunnelling but this is not true in the case of light nuclear fusion. The wave function will reflect back and forth inside the nuclear well.

The surprisingly good agreement between the theoretical calculation of fusion cross sections with experimental data implies that the compound nucleus model might not be applicable for the light-nuclei sub-barrier fusion. Instead the selective resonant tunnelling model is used. This provides a new approach toward nuclear fusion energy with no strong nuclear radiation for the sub-barrier fusion nuclear physics.

The good agreement between cross DD fusion section measurements and the theory $[12,13]$ is shown in Fig. 2. Any measurement of a DD fusion cross section for $470 \mathrm{eV}$ is far beyond the present experimental possibilities. We refer e.g. to the only theoretically concluded p-p weak force cross section which was not yet experimentally confirmed but its estimation fully explains [28] why the burning of protons at about 15Mill Kelvin in the centre of all the $10^{22}$ stars in the Universe takes few billion years. The theory [13] for DT at $470 \mathrm{eV}$ results in a fusion cross section of 


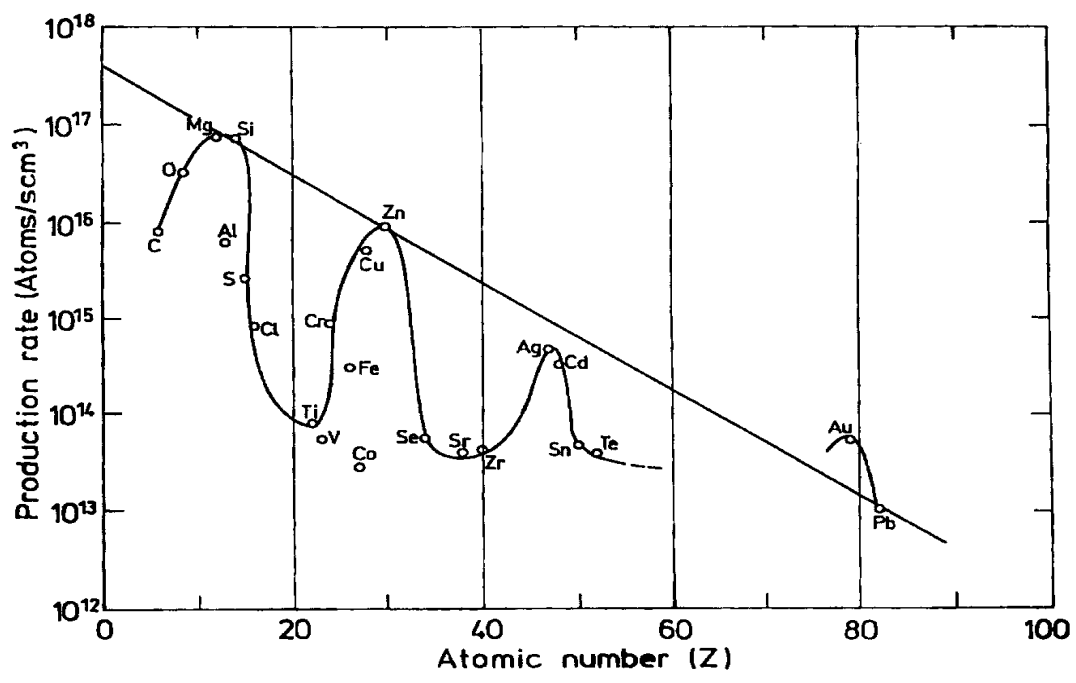

Fig. 3. Elements produced in palladium at very high concentrations of protons within several weeks of interactions by low energy nuclear reactions $[29$

$$
\sigma=3.6761 \times 10^{-26} \text { barns }
$$

which value is few orders of magnitude smaller than the estimated p-p weak interaction cross section near $1.5 \mathrm{keV}$. From the theory we can then conclude that the fusion reaction time of $U=10^{5} \mathrm{~s}$ for the pm distance DD reactions is comparable to the concluded $10^{25}$ times longer reaction time of the hot fusion DD reactions in fair agreement with the conclusion of the preceding section and of [22].

\section{5) Consistency Views}

This section is comparing some experimental results of nuclear transmutations, fission and combined nuclear reactions induced by protons incorporated mostly in palladium and nickel multi-layers as LENR (low energy nuclear reactions). We underline that these consideration may be taken as consistency proofs only and may support the existence of cold fusion and LENR only in a wider view than a direct proof would need.

Following the creation of a large range of elements during several weeks of interaction in a fully reproducible way with 18 runs in electrolytic experiments [29,30], there appeared a distribution of endothermic (nucleon number $A=60$ lager than iron) generation of nuclei with maxima close to the magic numbers, see Fig. 3. These production rates were given from the evaluation of SIMS (secondary ion mass spectrometry) measurements of the element distribution in the Pd-Ni layers before the electrolytic treatment, Fig. 4, and after the treatment, Fig. 5.

Despite an accurate analysis that the observed additional elements in the layers are 
not due to contamination from the electrolytic cell,the scepticism against all these kind of

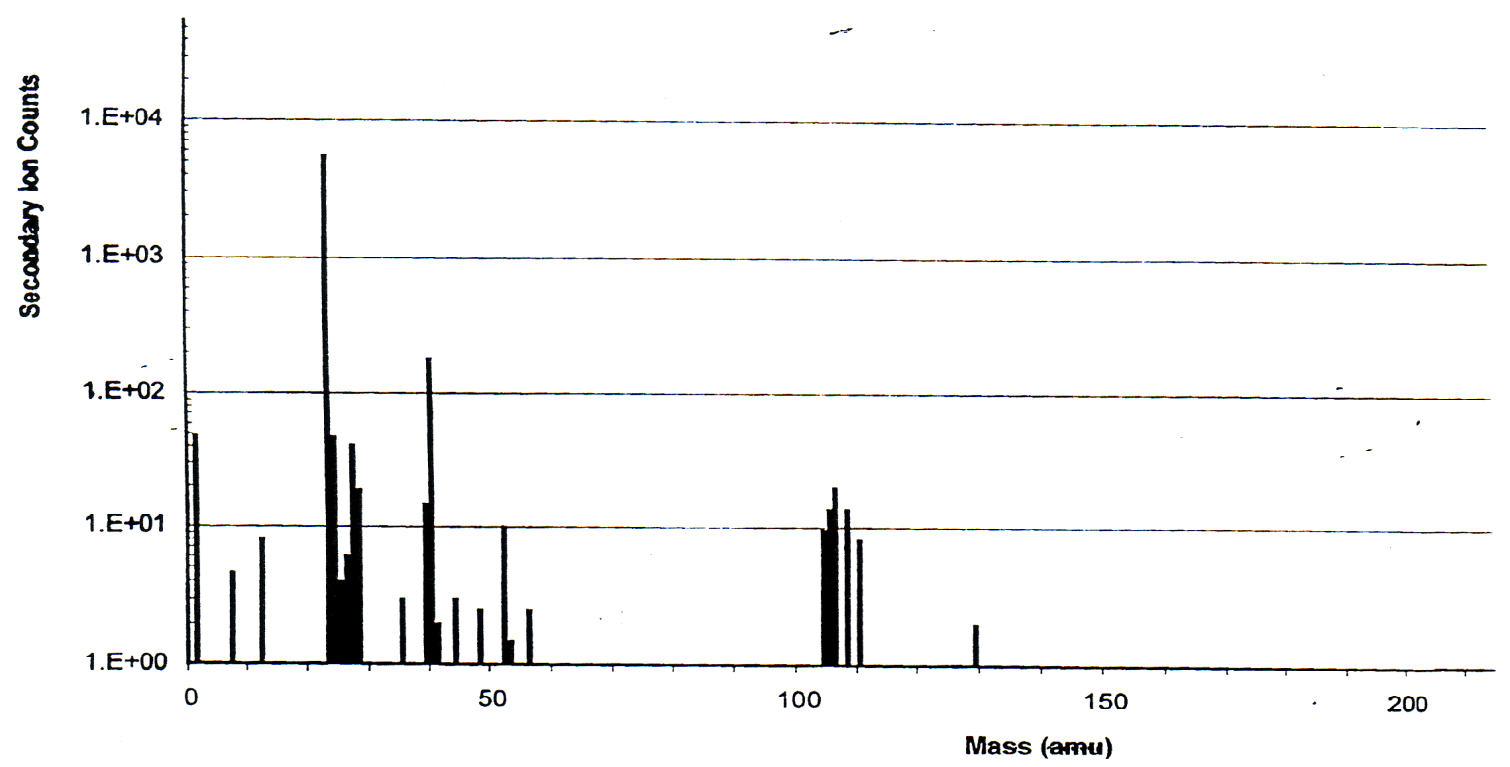

Fig.4. Element distribution from SIMS for Ni-Pd layers before reactions [29]

experiments is still there despite the reproducible results from 18 runs. We mentioned before the fact that rarest of rare elements (terbium) were uniquely measured [21] in other experiments which definitely could not come from pollution. The question was discussed [30] why these transmutation are not showing neutron, alpha or gamma emission and why not radioactive nuclei are produced via compound nuclear reactions for achieving the endothermic generation of heavy nuclei. The reason is that these reactions are slowly and there is time to find the most energetic branches in the compound reactions resulting in stable products different to the fast fission of heavy nuclei where one of the two daughter nuclei is radioactive. The fact that $\mathrm{MeV}$ energies of daughter nuclei are produced was seen from the larger than alpha traces in CR39 [19,20] and the emitted xrays in the $\mathrm{keV}$ range correspond to the bremsstrahlung of the daughter nuclei [20].

As proof in support of the reality of the LENR results, we show in this section how the results of Fig. 3 are consistent with general knowledge from other fields in physics.

\section{1) Endothermic production of nuclei and magic numbers}

Drawing a line through the maxima of the measure element distribution depending on the nuclear charge $\mathrm{Z}$ in Fig. 3, we find a Boltzmann-like probability distribution for the production rate $\mathrm{N}(\mathrm{Z})$

$$
\mathrm{N}(\mathrm{Z})=\mathrm{N}^{\prime} \exp \left(-\mathrm{Z} / \mathrm{Z}^{\prime}\right)
$$


where the best fit is with the decrement $Z^{\prime}=10$. This agrees with the element distribution in the Universe, Fig. 6, again with using the plot for the proton number $\mathrm{Z}$ in the nuclei based on the same $Z^{\prime}=10$.

The problem of the endothermic production of nuclei with $\mathrm{Z}$ above 26 (iron) is one of the unsolved important problems in astrophysics [31,32]. It should be noted that the

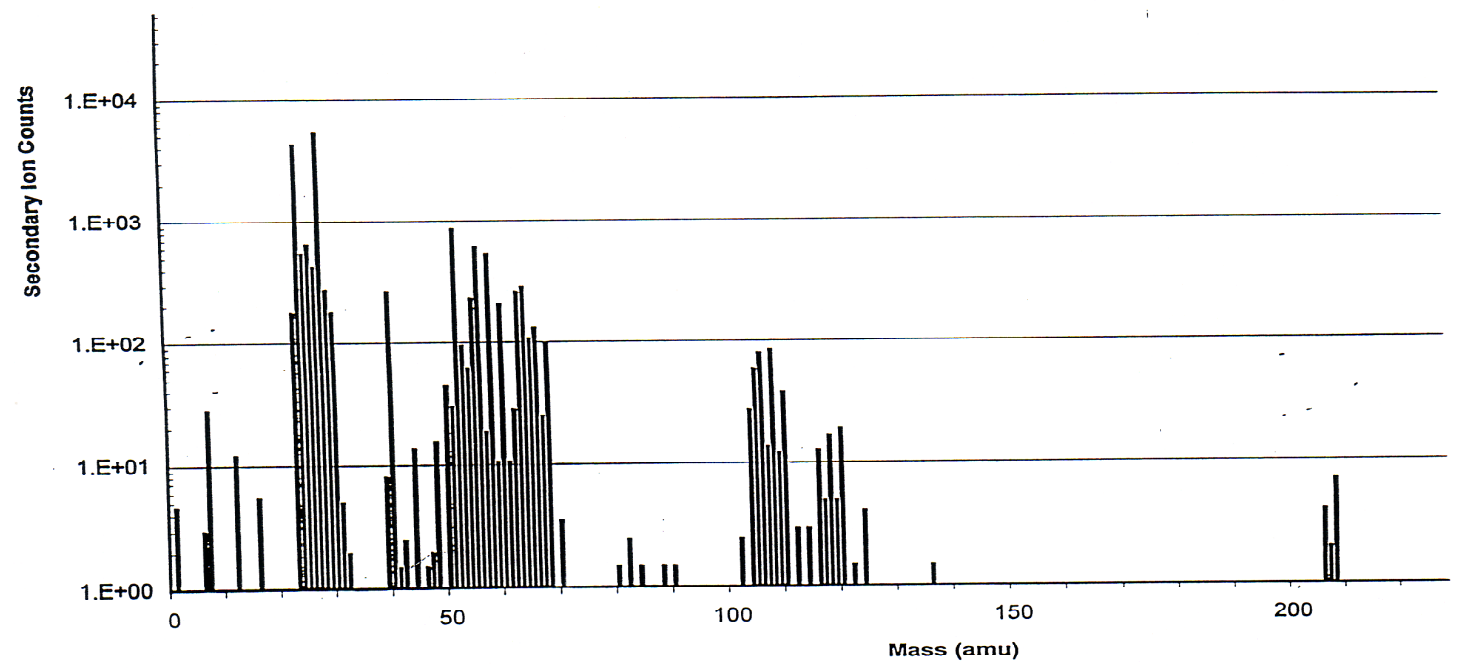

Fig. 5, SIMS mass spectrum after reactions [29]

distribution (5) only with $Z^{\prime}=10$ fits an interesting relation for the magic numbers of nuclei. We discuss here what consequences it has due to the fact that the drawn curve in Fig. 3 - fitting with the empirical astrophysical observations of the SAD - results in a $Z^{\prime}=10$ in Eq. (5) or values nearby. This is now related to the nuclear shell model where we derive an alternative foundation of the magic numbers compared to the usual explanation by spin and spin-orbit properties of nuclei. The magic numbers of the nuclear shell model are the sequence

$$
\text { magic numbers: } \mathrm{M}_{1} \in 2,8,20,28,50,82,126
$$

for protons $\mathrm{Z}$ in nuclides as well as for neutrons $\mathrm{N}=\mathrm{A}-\mathrm{Z}$ with the measured well known maxima of binding energies. We now calculate the ratios $R(n)$ for the astrophysical [30] SAD-Boltzmann probabilities from Eq. (5)

$$
\mathrm{R}(\mathrm{n})=\left[\mathrm{N}\left(\mathrm{Z}_{\mathrm{n}+1}\right) / \mathrm{N}\left(\mathrm{Z}_{\mathrm{n}}\right)\right]^{-1}=\exp \left[\left(\mathrm{Z}_{\mathrm{n}+1^{-}}-\mathrm{Z}_{\mathrm{n}}\right) / \mathrm{Z}^{\prime}\right]
$$

where the magic numbers $Z_{n}$ of the protons are taken with the following indices $n$ $(0,1,2,3 \ldots)$

$$
\mathrm{Z}_{\mathrm{O}}=2, \mathrm{Z}_{1}=8, \mathrm{Z}_{2}=20, \quad \text { for relation up to the magic number } 20
$$




$$
Z_{2}=28, Z_{3}=50, Z_{4}=82, Z_{5}=126 \quad \text { for the magic numbers above } 20
$$

As seen from Table 1 for $Z^{\prime}=10$ in Eq. (5), the ratios R, Eq. (7) result in values very close to

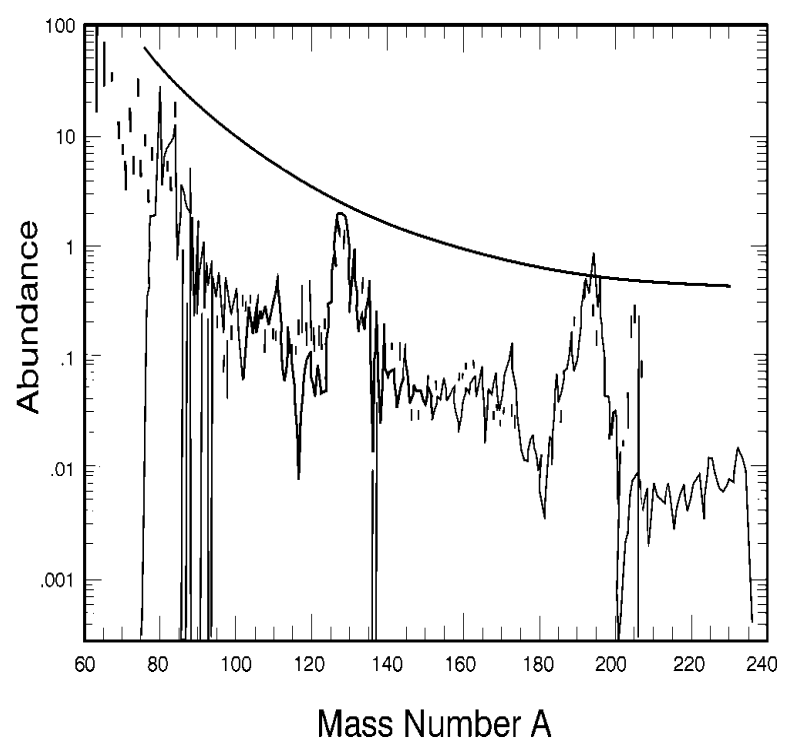

Fig. 6. Measured standard abundance distribution of the elements (SAD) [30] in the Universe where the line follows the exponential Boltzmann dependence of Eq. (5) with $Z^{\prime}=10$.

$$
\mathrm{R}(\mathrm{n})=3^{\mathrm{n}}
$$

shown in Fig. 7. The good fit with Z' $=10$ compared with other numbers can be seen for the magic number 81 at $n=4$. Instead of $R=81.45$ (being very close to $3^{4}$ for $Z^{\prime}=10$ ) we find $\mathrm{R}=224.69$ for $Z^{\prime}=8 ; \mathrm{R}=132.80$ for $Z^{\prime}=9, \mathrm{R}=54.598$ for $Z^{\prime}=11$.

Table 1. Sequence $n=0,1,2 \ldots$ of magic numbers with the values $\exp \left(Z_{n} / Z^{\prime}\right)$ and $R(n)=\exp \left[\left(Z_{n+1}-Z_{n}\right) / Z^{\prime}\right]$ of Eq. (7) with

\begin{tabular}{|c|c|c|c|c|}
\hline$n$ & Magic Number & $\exp \left(Z / Z^{\prime}\right)$ & $R(n)$ & $3^{n}$ \\
\hline 0 & 2 & 1.221 & 1.822 & 1 \\
\hline 1 & 8 & 2.2225 & 3.321 & 3 \\
\hline $2($ as $n+11 n$ & $\begin{array}{l}20 \\
28\end{array}$ & $\begin{array}{l}7.389 \\
12.1824\end{array}$ & 9025 & $\overline{9}$ \\
\hline $2(\operatorname{as~} n$ in $(8))$ & 50 & 148.413 & 24.53 & 27 \\
\hline 3 & 82 & $\begin{array}{l}3640.95 \\
2065585\end{array}$ & 81.45 & 81 \\
\hline $\begin{array}{l}4 \\
5\end{array}$ & & 296558.5 & & \\
\hline
\end{tabular}
$Z,=10$ from Eq. (5) as measured.

Extending the procedure with the $3^{\mathrm{n}}$-law (10) to higher magic numbers - see the extension of the fully drawn line by the dashed line in Fig. 7 [37] - one arrives at the following higher magic numbers indicated by open circles as closest values to the line. The result is that for $n=6$ one arrives at a magic number 180, for $n=7$ at 246 and for $n=$ 8 at 324 , 


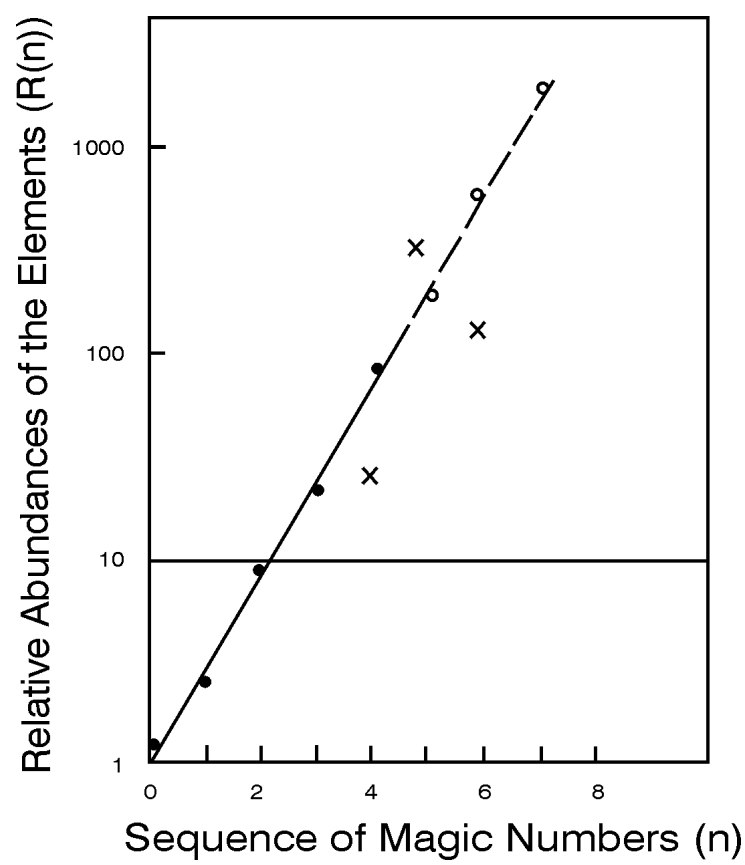

Fig.7. Values $R(n)=\exp \left[\left(Z_{n+1}-Z_{n}\right) / Z^{\prime}\right]$ for the sequence of magic numbers $n$ with specially defined exception of 20 and with the fitting value $Z^{\prime}=10$, (dots) compared with the $3^{n}$-relation (Eq. 10) [37] straight line. Circles are for derivation of new magic numbers (180; 246 and 324), Eq. (11) and crosses for earlier considered [30] numbers 114, 184 and 228.

shown by circles in Fig. 7. This can be compared with the earlier predicted magic numbers [33] 114, 184 and 228 (crosses in Fig. 7) which by far do not fit so well the relation (10).

The first conclusion of these results derived from this fitting of the Boltzmann probability (5) with the standard abundance distribution of the heavier elements observed in the Universe, Fig. 6, refers to the curious jumping procedure with the magic numbers 20 and 28 in Table 1 . This is exactly what was necessary to be explained when the magic numbers were discovered numerologically by Bagge. [34,35]. In order to explain the maximum binding energy of some nuclei, which can not be explained so easily as e.g. the electron shells in atoms from the Schrödinger equation with the well known $2 \mathrm{n}^{2}$-relation $(n=1,2,3 \ldots)$ for the electron shells, other estimates were needed. It is most remarkable that a purely speculative combination of the sequences $2 ; 3,4 ; 5 ; 6 ; \ldots$ and of the sequence 1 ; $2 ; 3 ; 4 ; 5 ; \ldots$. and their combinations [35] led Bagge [34] to the result of the following sequences (12) and (13) for the magic numbers. In the first case taking the sequence $2,3,4,5,6 \ldots$ as differences to produce $1,3,6,10,15,21 \ldots$ and then taking them as differences , one arrives at $0,1,4,10,20,25,56 \ldots$ and doubling these values,

$$
\mathrm{M}_{2} \in \mathbf{2 ,} \mathbf{8 , 2 0}, 40,70,112
$$


Beginning with the sequence $1,2,3,4,5,6, \ldots$ as the differences one arrives at $1,2,4,7,11,16$, $22 \ldots$ and again using these as differences leads to $0,1,3,7,14,25,41,63 \ldots$ which elements doubled leads to

$$
\mathrm{M}_{3} \in 2,6,14, \mathbf{2 8}, \mathbf{5 0}, \mathbf{8 2} . \mathbf{1 2 6}
$$

Bagge's question was why did the bold numbers fit the observed magic numbers and how to explain the jump from the Bagge sequence (13) to (14) after the first three elements. A well known explanation was given [35] by Jensen and Maria GoeppertMayer who noted that there is a difference in the spin and orbit configurations in the nuclei preferring in the one case the lower numbers of Eq. (12) and in the other case the higher numbers of Eq. (13).

In contrast to this explanation, we see now that the jump between the magic numbers 20 and 28 results systematically from the procedure of Table 1 without any need of a physical explanation of the spin etc.. If our explanation for a quark structure of the nuclear shells is the reason, this would be well different form the spin model and one has to learn again from a co-existence of basically different properties for the phenomena of the nucleus. Vice versa one may find an explanation of the spin-orbit phenomenon related to the threefold multiplicity of Eq. (10) concluding that the stable nuclear shells are combined each with three quark links of the higher shell to one quark in the low shell.

This consideration of the magic numbers was involved also to the generalization of the Debye length and the subsequent surface energy in laser produced plasmas to the degenerate electrons in a metal [25] with a subsequent quantum theory of surface tension of metals in agreement with measurements. Only instead of the temperature, the FermiDirac energy had to be used. Generalizing this furthermore to the Fermi energy of nucleons (protons and neutrons) the quantum surface energy is just compensating the internal energy - dominated by the Fermi energy - of the nucleons in a nucleus resulting at the measured size of nuclei. This is a new access to Bohr's droplet model of nuclei as it successfully explained the fission of uranium [36] - however - now on a basically new basis of the Debye lengths [38,39]. One further result is that at about six times higher than nuclear density, the change of the Fermi energy into its relativistic branch makes any nucleation impossible and results in a soup of particles where the mass is eliminated explaining then the quark gluon state. When expanding at the big bang from higher density to that of nuclei, the nucleation begins and the Boltzmann equilibrium permits the generation of nuclei including the endothermic nuclei, however only up to the atomic number of about uranium [39].

All these results are to some extend related to the reported measurements of LENR [29] and may indirectly link to a confirmation of work initiated by the cold fusion [9] whatever the motivations or some confusions to these initial developments had been.

References

[1] Brian Josephson, Pathological Disbelief, Lecture given at the Nobel Laureate Meeting, Lindau, June $30^{\text {th }}, 2004$ (Edited version, revised Aug. 20 ${ }^{\text {th }}, 2004$ )

[2] John Cockroft and A. Walton Proc. Roy. Soc. London A137, 229 (1933)

[3] Mark L.E. Oliphant and Lord Rutherford, Proc. Roy. Soc. London A 141, 259 (1933)

[4] M.L.E. Oliphant, P. Harteck and Lord Rutherford, Proc. Roy. Soc. London A144, 692 (1934)

[5] F. Paneth, Naturwissenschaften 14, 958 (1926)

[6] F. Paneth, Nature 119, 728 (1927) 
[7] O. Reifenschweiler, Internal Report Philips Research Laboratories 1961, see Phys. Letters A 184, 149 (1994)

[8] B.V. Derjaguin et al., Colloid J. USSR 48, 8 (1986)

[9] M. Fleischmann, S. Pons, J. Electroanalyt. Chem. 261, 301 (1989); M. McKubre, Proceedings of the $9^{\text {th }}$ International Conference on Cold Fusion, Beijing, May 2002, edited by Xing Zhong Li, (Tsinghua University Press, Beijing 2003) p. xviii

[10] S.E. Jones, Nature 338, 737 (1989)

[11] E. Crawford, R. Lewin Sime and Mark Walker, Physics Today 50 (No.9) 26 (1997)

[12] X.Z. Li, J.T. Tian, M.Y. Mei, C.X. Li, Phys. Rev. C 024610 (2000)

[13] X.Z. Li, B. liu, Q.M. Wei, H. Hora Laser and Particle Beams 22 No.4 (2004)

[14] H. Hora, Summary about Theory, Proceedings of the $9^{\text {th }}$ International Conference on Cold Fusion, Beijing, May 2002, edited by Xing Zhong Li, (Tsinghua University Press, Beijing 2003) p. xxi

[15] E. Yamaguchi, T. Nishioka, Nuclear fusion induced by the controlled out-transport of deuterions in palladium, Prof. $3^{\text {rd }}$ Intern. Conf. Cold Fusion, Nagoya, Oct. 1992

[16] M. Prelas, F. Boody, W. Gallaher, E. Leal-Quiros, D. Mencin, S. Taylor, J. Fusion Energy 9, 309 (1990)

[17] X.Z. Li, Bin Liu, Z. Ren et al, Proceedings of the $9^{\text {th }}$ International Conf. Cold Fusion, Beijing 2002, X.Z. Li ed. (Tsinghua Univ. Press 2003) p. 197

[18] J. Tian, B. Liu, X.Z. Li et al Proceedings of the $9^{\text {th }}$ International Conf. Cold Fusion, Beijing 2002, X.Z. Li ed. (Tsinghua Univ. Press 2003) p. 360

[19] A.G. Lipson, A.S. Roussetski, G.H. Miley, C.H. Castano, Proceedings of the $9^{\text {th }}$ International Conf. Cold Fusion, Beijing 2002, X.Z. Li ed. (Tsinghua Univ. Press 2003) p. 218

[20] Yan Deng, X.Z. Li, Conference three Gorges, 6 Oct. 1998

[21] X.Z. Li, S. Guang, Xiu M. Qiao, et al., Proceed. Asti Meeting Oct. 1999, W.F.M. Collins and R. George eds., Conference Proceedings (Italian Physical Society, Bologna 2000) p. 102

[22] H. Hora, J.C. Kelly, J.U. Patel, M.A. Prelas, G.H.. Miley,J.W. Tompkins Phys. Lett. A175, 138 (1993)

[23] Z. Hennis, S. Eliezer, A. Ziegler, J. Phys. G15, L219 (1989)

[24] J. Rafelski, M. Sawitzki et al fusion Technology 19, 136 (1991)

[25] H. Hora, Gu Min, S. Eliezer, P. Lalousis, R.S. Pease, H. Szichman, IEEE Trans. Plasma Science PS17, 290 (1989)

[26] S. Ichimaru, Rev. Mod. Physics 65, 255 (1994)

[27] R.G. Clark, H. Hora, P.S. Ray, Sir Ernest Titterton, Phys. Rev. C18, 1127 (1978)

[28] R. Kippenhahn, A. Weigert, Stellar Structure and Evolution (Springer, Heidelberg 1990)

[29] G.H. Miley, G. narne, M.J. Williams, J.A. Patterson, J. Nix, C. Cravens, H. Hora, Progress in New Hydrogen Energy, M. Okamoto ed., (New Energy and Industrial technology, Tokyo 1997) p. 627

[30] H. Hora, G. Miley and J. Kelly, Current Trends in International Fusion Research - Proceedings of the Third Symposium, E. Panarella ed. (NRC Press, National Research Council of Canada, Ottawa ON K1A 0R6, Canada 2002) . 527

[31] Michael Turner, see P. Gwynne, Physics World 14 (No.2) 6 (2001)

[32] Spencer Abraham, APS News 12 (No. 11) 8 (2003)

[33] M. Brack, P. Quentin, and D. Vautherin (1978) Proceedings of the International Symposium on Superheavy Elements, Lubbock, Texas, 1978, M.A.K. Lodla ed. (Pergamon, New York) p. 309; A. Sobieczewski, (1974) Phys. Scr.10A, 47; M. Schädel et al, Nature 388, 55 (1977); M. Schädel and J.V. Kratz, Physikalische Blätter 53, 865 (1997); P. Armbruster, Proceedings International Conference on Nuclear Physics, Florence/Italy 1983, P. Blasi and R. Ricchi eds., (Tipografia Compositori, Bologna 1984) p. 343, see also the agreement with the new magic numbers (12): K. Rutz, M. Bender, T. Bürenich, T. Schilling, P.G. Reinhard, J.A. Maruhn, and W. Greiner, Nuovo Cimento 110A, 1237 (1997)

[34] E. Bagge, Naturwissenschaften 35, 376 (1948)

[35] O. Haxel, J.H.D. Jensen and H.E. Suess, Zeitschr.f. Physik 128, 295 (1950)

[36] L. Meitner and O. Frisch, Nature 143, 239 (1939)

[37] H. Hora, Czechoslovak J. Physics 48, 321 (1998)

[38] H. Hora, Plasma Model for Surface Tension of Nuclei and the Phase Transition to the Quark Plasma, Report CERN-PS/DL-Note-91/05, August 1991, see also H. Hora, Laser Interaction and Related Plasma Phenomena (Plenum NY, 1992) Vol. 10, p. 19

[39] H. Hora, G. Miley, F. Osman and P. Hammerling, High Power Laser Ablation V, C.R. Phipps ed., SPIE Proceedings No. 5448 (2004) p. 119 